\title{
Corps, littérature, société (1789-1900), sous la direction de Jean-Marie Roulin
}

\section{Valentina Ponzetto}

\section{(2) OpenEdition}

1 Journals

\section{Edizione digitale}

URL: http://journals.openedition.org/studifrancesi/9245

DOI: $10.4000 /$ studifrancesi.9245

ISSN: 2421-5856

\section{Editore}

Rosenberg \& Sellier

\section{Edizione cartacea}

Data di pubblicazione: 1 juin 2008

Paginazione: 196-197

ISSN: 0039-2944

\section{Notizia bibliografica digitale}

Valentina Ponzetto, «Corps, littérature, société (1789-1900), sous la direction de Jean-Marie Roulin»,

Studi Francesi [Online], 154 (LII | I) | 2008, online dal , consultato il 10 janvier 2021. URL: http:// journals.openedition.org/studifrancesi/9245; DOI: https://doi.org/10.4000/studifrancesi.9245

Questo documento è stato generato automaticamente il 10 janvier 2021.

\section{(c) (i) $\odot$}

Studi Francesi è distribuita con Licenza Creative Commons Attribuzione - Non commerciale - Non opere derivate 4.0 Internazionale. 


\title{
Corps, littérature, société (1789-1900), sous la direction de Jean-Marie Roulin
}

\author{
Valentina Ponzetto
}

\section{NOTIZIA}

Corps, littérature, société (1789-1900), sous la direction de Jean-Marie Roulin, Presses de l'Université de Saint-Étienne, 2005, pp. 312.

1 Gli studi qui riuniti da Jean-Marie Roulin tratteggiano in modo vivido e originale il grande affresco di un xIX secolo ricco di conflitti e fermenti di rinnovamento attraverso una serie di rappresentazioni letterarie del corpo e della società, o meglio dei sottili e complessi rapporti che legano queste due tematiche, facendo di una lo specchio dell'altra. Come ben nota il curatore, nel nuovo mondo emerso dalla Rivoluzione francese, che ha dotato il cittadino di un nuovo corpo giuridico, politico e sociale, «écrire le corps, c'est interroger les signes d'une appartenance sociale ou politique, scruter les traces qu'a pu y laisser l'Histoire, sonder la manière dont la loi agit sur l'individu». La rappresentazione letteraria del corpo si carica dunque di segni che rivelano i rapporti dell'individuo con il contesto storico e socio-politico in cui vive. Il volume si articola in quattro parti, improntate ad altrettante prospettive di ricerca.

2 La prima, «Le Discours politique du corps», si apre in modo simbolico sull'articolazione fra la Rivoluzione, evento fondatore del periodo studiato, e il teatro, spazio della rappresentazione per eccellenza. In «théâtraliser la Révolution française» (pp. 31-40), Pierre FRANTZ mostra come durante la Rivoluzione la politica sia fortemente teatralizzata attraverso messe in scena rituali e spettacolari, in cui la corporeità gioca un ruolo importante: dalle esecuzioni sulla ghigliottina, alle feste repubblicane, dove gli attori sono chiamati ad incarnare figure allegoriche come la Libertà o la Ragione, ai solenni omaggi funebri resi a grandi personaggi come Marat. Ancora al teatro come 
luogo privilegiato dove esplorare problematiche politiche è consacrato l'articolo di Lucienne FRAPPIER-MAZUR, L'art du comédien comme métaphore du politique: "Pierre qui roule" et "Le Beau Laurence" de Gorge Sand (1869), (pp.41-54). Lo studio del dittico sandiano rivela come per la romanziera la troupe teatrale sia una sorta di metafora della società e il luogo dove sperimentare una piccola repubblica utopica. La compagnia è pensata come un corpo, alla maniera della Repubblica nei discorsi politici della Rivoluzione. Il valore metaforico del corpo è invece negato e demolito da Sade, come si può leggere nell'intervento di Jean-Christophe ABRAMOVICI, Sade et les corps fantômes (pp.55-63). Impotente a raffigurare la politica, il corpo sadiano segue tuttavia un modello politico, segnatamente nella distinzione fra la sessualità cerebrale degli aristocratici libertini e quella vitale e inesauribile dei fouteurs plebei. Nel Comte de Monte-Cristo di Dumas, studiato da Sara MOMBERT (L'enfermement et la métamorphose. La politique au miroir du corps, pp. 65-78), le vicende fisiche dei personaggi si fanno portatrici di una critica della società. Alla reclusione, immagine dell'immobilismo sociale della Restaurazione, si oppongono le continue metamorfosi dell'eroe, che attraverso la manipolazione delle apparenze può infine affermare la propria libertà. Analogamente, in La marque, la lettre, le sexe: le corps de Vautrin (pp. 79-88) Pierre LAFORGUE dipinge il personaggio balzacchiano come vivente incarnazione di un conflitto fra la legge, iscritta a forza nella carne attraverso il marchio del forzato, e l'individuo che la contesta e la elude.

3 La seconda sezione del volume, "Corps du Christ, corps du roi, corps du peuple», interroga il difficile transfert, anch'esso originato con la Rivoluzione, dal corpo sacralizzato del re a quello del popolo e della Nazione, fra nostalgie dell'immaginario monarchico e riaffiorare del dualismo cristiano. Al nuovo Cristo dei sansimoniani, incarnato nella controversa figura del Père Enfantin, fra riabilitazione della carne e teologia politica, è consacrato il saggio di Philippe RÉGNIER, Corps du Roi, corps du Christ: la personne symbolique et les théories charnelles du Père Enfantin, chef suprême de l'Eglise saintsimonienne (pp.91-108). Il corpo dei sovrani riappare invece nelle ricostruzioni del passato. Eliane VIENNOT si volge al romanzo storico, nella fattispecie La Reine Margot di Dumas, dimostrando come la rappresentazione degli ultimi Valois e le deformazioni a cui è sottoposta la realtà storica siano qui volti a giustificare il rigido ordine dei ruoli maschili e femminili così come imposto dalla società ottocentesca (Le corps signifiant des souverains, pp. 109-125). L'opera storica di Michelet cerca invece di demolire, una volta per tutte, la fascinazione per il corpo del re e l'aura mistica e simbolica che lo circonda. È quanto viene messo in evidenza dall'articolo di Paule PETITIER, 'L'anus du Roi Soleil'. Le corps dans l'“Histoire de France au XVII ${ }^{e}$ siècle de Michelet (pp. 127-140), che segue lo storico nella sua descrizione desacralizzante dei regali acciacchi e nella sua caccia ai suoi propri fantasmi. Lo studio di Corinne SAMINADAYAR-PERRIN sul ciclo di Dumas dedicato alla Rivoluzione fa luce sulla difficile, se non impossibile, traslazione di una sovranità politica dalla monarchia decaduta al popolo, incapace di dar corpo a una nuova nazione (L'insaisissable corps du peuple dans le cycle révolutionnaire de Dumas, pp. 141-160).

4 «Les incarnations de l'Histoire, da cui trae il titolo la terza parte, sono quelle che i romanzieri traducono nelle figure dei loro personaggi, variamente segnati dalla traccia degli eventi. Les 'amants de la mort': physionomie du corps révolutionnaire dans les 'Mémoires d'outre-tombe' de Chateaubriand di Agnès VARLET (pp.163-173) analizza le funzioni e i significati che assumono per lo scrittore le metafore del corpo nel racconto della caduta della monarchia e nei ritratti del popolo o dei capi della Rivoluzione. Simbolizzazione del corpo dell'individuo e parallela de-simbolizzazione del corpo del sovrano sono al 
centro dello studio di Stefano LAZZARIN (Balzac et le corps des nobles: à propos du BCabinet des Antiques', pp. 176-189) che propone, attraverso una galleria di personaggi balzacchiani, dei corpi da leggersi come altrettante metafore della situazione politica postrivoluzionaria. La maniera in cui la Storia agisce sui corpi e l'ideologia si inscrive nella carne oltraggiata e sofferente dei personaggi in Madame Putiphar di Pétrus Borel è l'oggetto dello studio di François KERLOUÉGAN, 'Madame Putiphar' di Pétrus Borel: historicisation du corps et incarnation de l'histoire (pp. 191-200). L'onnipresenza del corpo per tradurre una visione della storia si ritrova anche in 'Le Chevalier Des Touches' ou l'Histoire incarnée (pp. 201-214), in cui Xavier BOURDENET passa in rassegna tre tipologie di corpo nel romanzo storico di Barbey d'Aurevilly: «corps grotesque, corps en gloire e corps sanglant». Infine anche Philippe DoufouR (L'histoire à fleur de peau, pp. 215-237) si interessa alla scrittura del corpo nel romanzo storico. Da Walter Scott al Flaubert di Salammbô, passando per Hugo, Dumas, Balzac e Dickens, il critico mostra come la descrizione fisica subisca spesso, nell'Ottocento, un trattamento allegorico.

5 L'attenzione per la pratica della scrittura che è venuta vieppiù disegnandosi nel corso del volume, costituisce l'oggetto principale della quarta parte, «Corps et création», che coinvolge in ultima analisi lo scrittore in persona. Stéphane CHAUDIER analizza la poetica dell'allegoria nelle Fleurs $d u$ mal, fra tradizione cristiana dell'incarnazione e protesta politica ('Ô blasphème de l'art!': Baudelaire et les allégories déconstruites, pp. 241-251). Christine PLANTÉ si interessa al posto dello scrittore nello spazio pubblico attraverso un caso esemplare, quello di Gorge Sand, particolarmente in vista in quanto celebre scrittrice e in quanto donna. 'Signes particuliers: aucun'. Le corps de l'autobiographe dans "Histoire de ma vie" de George Sand (pp. 253-272) ci dice come l'autoritratto della romanziera si costruisca attraverso lo sguardo altrui, ma anche come inviti il lettore al riconoscimento e all'identificazione: ritratto di una generazione piuttosto che autoritratto. Una paradossale rappresentazione dello scrittore e della sua capacità di concepire e creare è dipinta da Philippe BERTHIER nella sua analisi di Mémoires de deux jeunes mariées (Accoucher au masculin, pp. 293-305) in cui Balzac, descrivendo la vita delle due protagoniste e in particolare l'esperienza della maternità e del parto, arriva a calarsi perfettamente nei panni di una donna, come se l'artista non potesse pensare la sua creazione che attraverso l'immagine del corpo femminile. Il percorso del secolo volge al termine con l'intervento di Yoan VéRILHAC: Le corps de l'écrivain dans le discours de la jeune critique fin de siècle (1885-1900), (pp. 273-292) che passa in rassegna ritratti di scrittori decadenti, apparsi sui periodici fin de siècle dopo la significativa rottura segnata da À Rebours, per mostrare come il corpo diventi uno dei vettori principali della riflessione sul ruolo dello scrittore nella società e sul suo rapporto con il potere letterario e politico. 